\title{
Reflections on the Present Situation of Social Platform Operations of Chinese Professional Basketball Sports Clubs:
} A Case Study of CBA Guangdong South China Tigers Club

\author{
Xuanzheng Lin*
}

Malvern College QingDao, Qingdao, 266109,China
Corresponding Author's Email:1164467201@qq.com

\begin{abstract}
With the advent of social media age, professional basketball clubs are paying more and more attention to the operation of their official social media. This paper takes Guangdong South China Tiger Club, the most successful CBA team, as an example, and analyzes $317 \mathrm{WeChat}$ articles, 54 TikTok videos and 3558 Weibo contents published by the club through text analysis. According to the research, the Tigers pursues matrix building in social media operation, mostly focusing on highlights of matches on WeChat official account (OA), text live broadcast on Weibo, and introduction of players' daily life in off-season on TikTok. The shortcomings are less fan interaction, content of single style and slow update speed. In this case, this paper argues that the Tigers can increase the interaction with fans, speed up the update speed of videos and articles, strengthen its ability in real-time text live broadcast and other aspects of social media operation, and focus more on the contents that fans want to see and the daily life of players that fans are concerned about.
\end{abstract}

Keywords: Guangdong South China Tigers Club, social media, operation and maintenance, fan interaction.

\section{INTRODUCTION}

In the social media age, professional clubs are paying more and more attention to the development of their own official social media platforms. As a professional league with high professionalization, the teams in the CBA league embarked on their exploration on this front a few years ago. Among them, Guangdong has been recognized as the most top team in the CBA because of its 11 titles and the 3-peats this year. However, compared to the excellence in competitions, the Tigers, subject to a few problems, are not doing very well in the social media platforms.

As a matter of fact, there are researchers who have been involved in the social platform operation of professional clubs for a long time. However, there are very few related studies regarding domestic basketball clubs. I only manage to identify two influential studies. Due to the time constraint, the above-mentioned literature only covers the team's operation on WeChat official accounts, but not other social media such as Weibo and Tiktok. It is inevitable that there are some partial generalizations and a lack of good understanding of all of its social media operation. Of course, this also leaves room for further research in this paper.

On the basis, this paper takes Guangdong South China Tiger Club, the most successful CBA team, as an example, and employs text analysis to examine 317 WeChat articles, 54 Tiktok videos and 3558 Weibo contents published by the club, in an attempt to find out the current characteristics and shortcomings of the Tigers on social platforms and make corresponding suggestions for improvement.

\section{ANALYSIS OF THE CURRENT SITUATION OF THE TIGERS' SOCIAL MEDIA PLATFORM}

The Tigers established an official account in Weibo as early as 2015, and opened its own WeChat OA and Tiktok account in 2016 and 2018, initially establishing its own social platform propagation matrix. The operation and maintenance of the three social media platforms have attracted not only fans but also those unfamiliar with basketball, increasing fans of the Tigers to a large extent. So, how exactly does the Tigers run its 
social media platforms, and what kind of characteristics does the media content on different platforms have?

\subsection{Match Summaries and Exciting Video Clips Dominate in the WeChat $O A$}

On the text, the content of CBA Tigers' WeChat OA is mainly divided into text, pictures, videos, music and prizes, and the home page of the push content is often like the "Graphic News Digest" , which generally uses pictures and a title for the headlines, and other content is attached in the form of headline links. The CBA is held across years, with preseason games generally held in October and regular season and playoff games held from November to next April.

During the CBA league games, the permanent content of the Tigers' OA includes game broadcast, five best plays, player interview, quiz show, etc. It summarizes the game in medium-length text, highlighting the most exciting part of the game coupled with the player's data. The WeChat operator specifically arranged personnel to work at home location of the club in Dongguan. As WeChat content producers and administrators, they can ensure frequent and close access to the players. Once the game is over, they are able to conduct timely interviews with the players and publish some short text or video reports with flash quotes through WeChat OA in the first place.

In the off-season after the league, the account mainly keeps track of the players in the holidays, reports second team and third team news, historical review, players' off-season entertainment news and other contents. During this period, Dongguan team WeChat OA focused on players' holiday trends. For example, it releases tracking reports on Zhao Rui, Yi Jianlian, Xu Jie, Hu Mingxuan and other players participating in the training of Chinese men's basketball training team. The dynamic reports of the second and third teams are also essential contents of the WeChat OA, such as the news of Guangdong Hongyuan Southern Tigers participating in the national U17 and U19 men's basketball youth league. In order to keep the public attention on the Tigers, WeChat public number also planned to report some historical review of the team and star players' performance in the league by New Century News, adding some soft news, for example, "Your friend Zhao Rui is online"; "Justin's debut", etc. in the 2016 - 2017 season.

\subsection{Textual Reports in Weibo}

Up to now, the Tigers have published 3588 microblogs, of which more than 2500 are related to text reports. Specifically, whenever there is a match, the Weibo account of the Tigers will always be the first to broadcast live text, timely broadcast of the dynamic developments on the field, so that fans can quickly understand the rapid changes on the court. In addition to the live text reports during the game, the post-game technical stats are also updated in a comprehensive and timely manner. When fans are unable to watch the game, they can follow the main information of the game with the text reports on Weibo merely. In addition, where there is no game, the Tigers Weibo account is dominated by tweets about the team's reunion and preparations, about volunteer activities the players participate in, and about retweets of domestic and international basketball information (especially official CBA League tournament). Strangely enough, as the official account, The Tigers never post texts, photos or videos related to the team or individuals.

\subsection{Players' Daily Lives in Off-season Are Introduced in TikTok}

Compared with Weibo and WeChat, the operation of its TikTok account started late. Among the 54 works visible so far, we found that 39 of them are related to the players' daily life, accounting for $86.7 \%$ of the total content. Such proportion is very different from the content pushed by WeChat and Weibo account. If Weibo and WeChat focuses more on the players' performance on the field, while TikTok highlights their life off the field. Be they players' training routine or video clips of related activities, another side of Tiger players in their daily life is presented: on February 24, 2020, a video of Hu Mingxuan's daily strength training was released; on July 30, 2020, a garbage classification advertisement inviting Zhao Rui, Du Runwang and Xu Jie was released.

\section{OPERATION SHORTCOMINGS}

Through the above analysis, we can already realize the current situation of the Tigers' operation on the three social media platforms. If analyzing a bit, it is easy to find that although the Tigers has gradually normalized its operation on social media site and its own characteristics have come into being, there is still a lot of room for improvement. The author believes that at present the main problems of the Tigers in developing social media platforms are: few fan interaction, content of relatively single style, slow update speed.

\subsection{Few Fan Interactions}

Insufficient interaction with "fans" is the most significant problem of the Tigers on various social platforms. For example, on TikTok, the Tigers released 54 videos, which received $192.3 \mathrm{w}$ likes and $37.1 \mathrm{w}$ fans, and the number of likes and comments of each video reached 10,000 and above, but the official TikTok operator only replied to 1 person in total, and basically did not reply to most of the fans' comments.

In fact, operation staff of the WeiChat OA who are responsible for the editorial work, lived in Dongguan, 
which enables them to get the first-hand news thanks to the proximity. The value of WeChat is realized through interaction, and the increase in interaction is to increase followers and improve communication with users. Although there are interactions during league games such as quiz show, in general, the Tigers only regard WeChat as a tool to deliver information, and do not leverage their advantages of all-day interaction, basically no interactive activities with "fans", which inevitably reduces the motives of fans to follow the account.

On Weibo, With 1.41 million followers and an average daily reading volume of $1000+$, the interaction between the Tigers and fans is also not very optimistic. According to the author's examination of the current 3558 microblogs, I found that the official microblogs rarely respond to fan messages.

\subsection{Content of a Single Style and Serious Homogenization}

During the league, the reports of the Tigers are predominantly match developments, while more detailed text or videos are available seen on Sina, Tencent, Netease and other portals. In comparison, the Tigers have almost no exclusive and unique match reports and do not pay enough attention to the players' life.[4] In addition to their performance on the court, fans are also interested in what the players are like off the court when they are beyond the reach. For the moment, except for the off-season training and commercial shooting of the players on Tiktok, and the sporadic messages about players' diet on WeChat OA, there is basically no information that can easily attract public attention such as team culture development and the interview feature articles of players.

\subsection{Slow Update Speed}

In the social media age, the speed and frequency of information change is increasing. People's demand for information grows with each passing day. However, the Tigers have failed to develop countermeasures on social media platforms. Specifically, of the 54 videos posted on Tiktok, the last one was posted on February 17, 2021, a quarter from now, which undoubtedly greatly reduces the attention, the number of followers and team popularity. On WeChat, we found that during the march of the season, it was basically a 3-day push, but that frequency immediately dropped to 1 update a month by the offseason. This update speed is obviously a big gap compared to the fans' expectations. In addition, compared to TikTok and WeChat, Weibo is relatively fast in updating content. As the content is mostly re-posted CBA news, it cannot meet the fans' expectation for quick access to diversified information.

\section{COUNTERMEASURES}

Given the current shortcomings, the author reckons that if the Tigers want to better its social media operation, thus further improving the popularity of the club brand, it can make efforts on the following aspects.

\subsection{More Fan interaction}

The Guangdong South China Tigers Club can give proper responses to readers and fans on WeChat OA. At present, among the nearly 100 released public messages, the team has replied 0 message to the fans, which will greatly reduce the fans' passion for the Club. On TikTok, In terms of TikTok, the Club has only replied to 0 video out of 54, and the interaction with fans is also basically 0 . Among the 3,558 contents released on Weibo, the interaction with fans is also 0 . This will undoubtedly frustrate the fans' feelings towards the team. Therefore, I think the Tigers should appropriately increase the interaction with fans on these three platforms, so that more people will pay attention to the Guangdong team, such as more gifts, basketball related equipment lottery and other activities. This will not only build a bridge of interaction between fans and the team, but also facilitate the team's operation commercially such as cultural and creative products. Only when more fans are more willing to leave messages under the platform and more fans feel the respect and recognition of the team, more people will pay attention to Guangdong South China Tigers Club and the brand image and cultural value of the club will become better.[1]

\subsection{More diverse content}

First of all, the present content of team WeChat OA is predominantly post-game summaries and highlights of the match. The content of single style and long-term lack of novel ideas to attract fans will inevitably bore the public and may even take a toll on continuous attention to the team. Weibo has the same story. The content of Weibo is mainly post-game analysis or re-tweeting Chinese news or CBA news, which makes the original single Weibo content even more boring. In terms of TikTok, as mentioned above, there basically single videos with no novelty to speak of. It neither updates regularly nor employs the latest features to attract the public's attention to the team.

Based on this, I think maybe we can differentiate the push content for three different social platforms, namely WeChat OA, Weibo and TikTok, so as to improve the content diversity. For example, on WeChat OA, we can add game previews, in-depth player interviews, feature stories, etc.; on Weibo, we can set more topics related to the team or basketball to attract fans to participate in the topics, so as to strengthen the interaction with fans; on TikTok, we can appropriately add funny or daily videos of players, as well as more player advice, Top five Plays, 
off-season player status, etc. In addition, considering that what attracts fans nowadays may not only be basketball skills and tactics itself, but also some basketball equipment, even the beauty and shape of some athletes, as well as entertaining and funny content, are also important reasons for them to pay attention to basketball. Therefore, if we can post this kind of content in different social platforms, it may not only help to increase the number of fans of the team and the number of video plays, but also may make more people pay attention to the Tigers.[3]

\subsection{Speeding up updates}

As mentioned above, it is obvious that the update frequency of the Tigers on social media platform is already difficult to meet the needs and expectations of Tigers fans. Therefore, speeding up update is obviously an important issue that the needs to be solved urgently. Considering that the players need time to rest and adjust, Tiktok could do weekly or 3 days update to keep the fans' attention on the Tigers. In terms of WeChat OA, a weekly update is suggested, so that the fans want to pay attention to the content and feel that WeChat OA content seems less lacking. As for Weibo, a daily update may be more sustainable to attract the attention of fans. After all, Weibo is the most mainstream public discussion platform in China today, and the team can explore a lot of original, re-tweeted and commented content on a daily basis. As long as the club's operation staff are good at discovering topics, there is little difficulty in achieving the vision of a daily update.[2]

\section{CONCLUSION}

The brand value of a sports club is not only reflected in the competitive field, but also in all aspects of business operation and fan culture. Among these, the role of social media obviously is never inconsequential. However, through a case study of the most successful CBA team, the Tigers, this paper finds that the development and maintenance of social media platforms of basketball clubs in China still have shortcomings such as few fan interaction, content of single style and slow update speed. [1]This is obviously not conducive to the branding and cultural deposit of Chinese professional basketball clubs. Therefore, in this sense, the research is not only a case study of the Tigers, but also a reminder for other teams to focus on the development of their own social platforms in addition to the competition itself, so that social media can become an important driving force for the development of the team.

\section{REFERENCES}

[1] Li zhengliang, And Wang Junyu." How professional sports Organizations improve social media audience Interaction: Based on the content analysis of NBA and CBA official Weibo." Press 23(2016):48-55.

[2] Feng Qi, And Luo Xuan." Social Media Communication Analysis Of Sino-German Football Clubs." Sports Culture Guide 000.007(2014):122-125.

[3] Zhang Desheng, Zhou Jie, And Li Feng." The Application Of Wechat Platform In The Brand Communication Of Professional Sports Club: A Case Study Of Cba Dongguan New Century Club." Journal Of Guangzhou University Of Sport 036.001(2016):7-10.

[4] Liu Di. Study on the Operation situation and Countermeasures of Jiangsu Kendia Professional Basketball Club. Diss. Beijing Sport University, 2017. 